\title{
Propeller blade signatures in the wavelet domain
}

\author{
D. H. Smith*
}

(Received 11 November 2004, revised 8 February 2005)

\begin{abstract}
An in-flight airframe vibration data sample taken from a propeller driven cargo aircraft is viewed in the wavelet and frequency domains, with an emphasis on propeller blade activity. Close examination in the wavelet domain reveals a mechanism by which this activity is shared between adjacent resolution levels, with subsequent wavelet packet decomposition demonstrating alternatives with fewer basis functions.
\end{abstract}

\section{Contents}

\section{Introduction}

${ }^{*}$ DSTO Edinburgh, PO Box 1500, Edinburgh, South Australia 5111. mailto:david.h.smith@csiro.au

See http://anziamj.austms.org.au/V46/CTAC2004/Smit for this article, (c) Austral. Mathematical Soc. 2005. Published 15 March 2005, amended March 18, 2005. ISSN $1446-8735$ 
2 Data in the time and frequency domains

C78

3 The discrete wavelet transform

C78

3.1 Coefficient/energy spectra and level components . . . . . .

$\mathrm{C} 79$

3.2 Propeller blade signatures . . . . . . . . . . . . . . C81

3.3 Wavelet packet decomposition . . . . . . . . . . C86

4 Summary and conclusions

C87

References

C88

\section{Introduction}

Transforms of measured time series data reveal structure and behaviour that may not be immediately apparent in the original data domain. Application of the ubiquitous discrete Fourier transform [1] involves switching entirely to the frequency domain and losing all temporal resolution in a single step. Discrete wavelet transforms reside in the intermediate region between these two extremes, possessing a compromise between time and frequency resolution drawn from a rich variety of possibilities [8]. The objective of this study is to examine an in-flight airframe vibration data sample in a set of different wavelet bases to gain basic understanding and insight into the mutual interaction between data and transform, with complementary frequency domain analysis providing a useful contrast. A focus on propeller blade activity shows a certain sharing mechanism taking place between a pair of adjacent wavelet resolution levels, or scales, which undergoes a particular behaviour pattern as the wavelet basis is changed. Application of the wavelet packet decomposition, as used on helicopter vibration data to focus on spectral regions for the main and tail rotors [5], provides an alternative propeller blade signature employing fewer basis functions. 

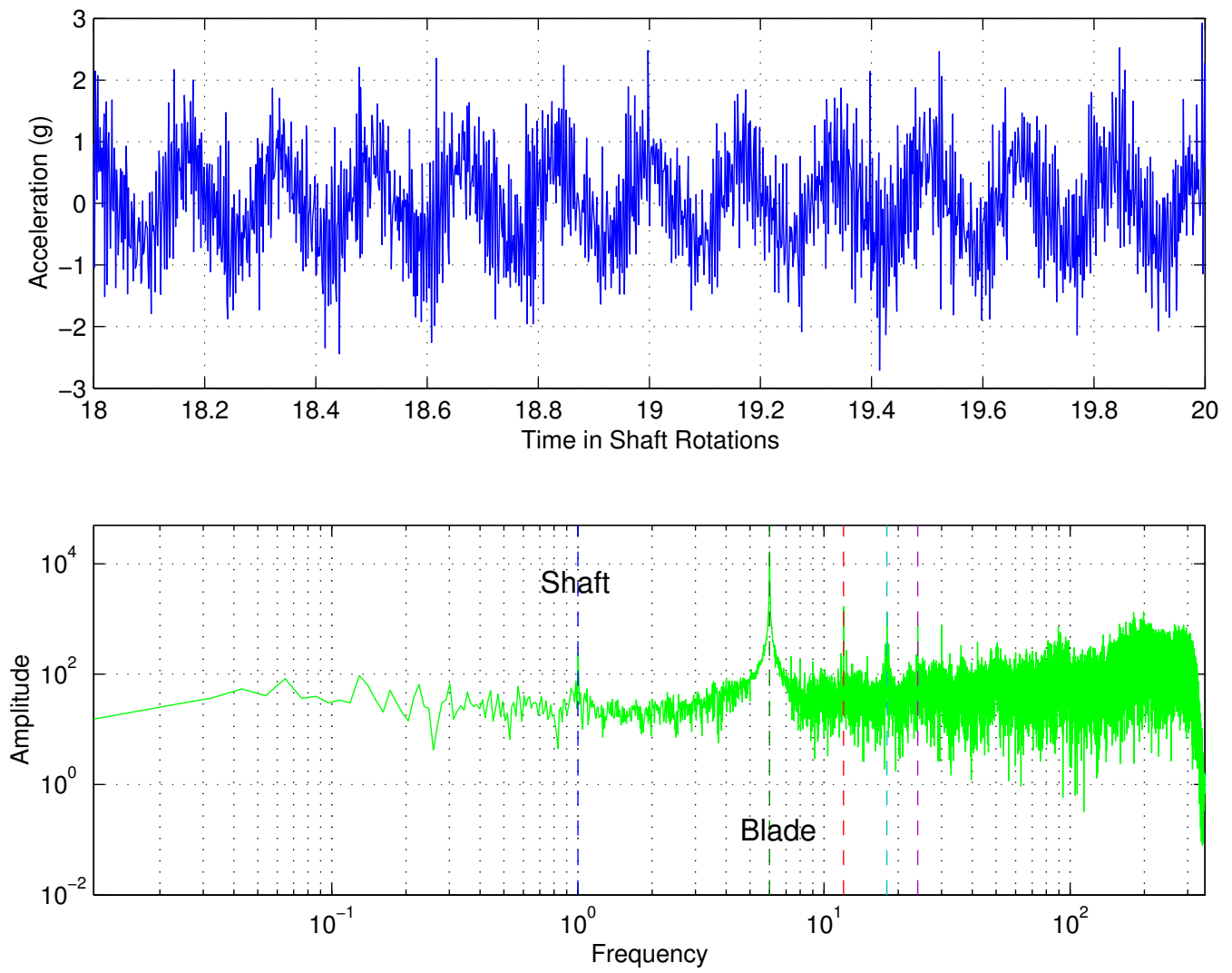

FiguRE 1: Flight vibration data segment comprising two propeller shaft rotations in the time domain, and a frequency domain representation for $2^{16}$ data points spanning 93 shaft rotations containing this particular segment. Periodic activity at six times the shaft frequency, representing the propeller blade motion, is visible in both domains. 


\section{Data in the time and frequency domains}

The data under consideration comprises a time series of $2^{16}=65536$ airframe accelerometer measurements taken near the propeller plane of a C130J cargo aircraft under high altitude flight conditions at constant airspeed [4], constituting just under 93 propeller shaft rotations. A segment containing two rotations is shown in Figure 1 together with a discrete Fourier spectrum calculated from the full series. Periodic activity representing the six propeller blades is clearly visible in the time domain picture, also showing itself as a dominant peak in the frequency domain, accompanied by additional peaks at higher multiples of the shaft frequency suggesting superharmonic behaviour [7]. Superimposed on the periodic component is an irregular rapid transient spike behaviour responsible for a considerable amount of energy in the high frequency region, the final segment of which, above 100 times the shaft frequency, contains over two-thirds of the total signal energy content. Final rapid decay visible above 300 is a result of analog filtering applied as an anti-aliasing measure. While this particular view captures the essential frequency content of the signal, it says little about the transient activity taking place on the basic blade component apart from its high frequency content.

\section{The discrete wavelet transform}

The time and frequency domain data representations display two extremes of sparsity, the former case characterised by the identity matrix and the latter characterised by a full matrix comprising basis vectors spanning the entire data interval. Wavelet bases reside in between these extremes, possessing a compromise between time and frequency resolution. A discrete wavelet transform provides a representation of the data vector $\mathbf{y}$ in terms of a basis 
comprising wavelets $\psi_{j k}$ on a set of resolution levels denoted by the index $j$

$$
\mathbf{y}=\sum_{k=0}^{2^{J}-1} \lambda_{J, k} \varphi_{J, k}+\sum_{j=J}^{n-1} \sum_{k=0}^{2^{j}-1} \gamma_{j, k} \psi_{j, k},
$$

and scaling functions $\varphi_{J k}$, which make up a reference signal on the bottom resolution level $J$. In matrix form, (1) constitutes the inverse transform

$$
\mathbf{y}=W \mathbf{g}
$$

where the first $2^{J}$ columns of $W$ contain the scaling functions $\varphi_{J k}$, with wavelets $\psi_{j k}$ making up the remaining columns in order of increasing resolution $j$ and $\mathbf{g}$ holding the associated coefficients. Forward transformation proceeds by the recursive application of high and low pass filtering operations, generating "approximations" and "details" at every stage [8]. For the Daubechies family of orthonormal, compactly supported wavelets under consideration, individual members $D_{N}$ are labelled by an integer $N$, according to its number of vanishing moments, which bears an intimate connection with its support width, regularity and frequency resolution properties [3].

\subsection{Coefficient/energy spectra and level components}

A $D_{2}$ wavelet coefficient spectrum, with modified boundary filtering applied [2], for this sample is given in Figure 2, in which the coefficients are arranged level by level in vertical bands, each of which represents the entire data interval on a particular scale. Resolution level $j$ comprises $2^{j}$ individual wavelets $\psi_{j k}$, where $k=0, \ldots, 2^{j}-1$ is the translation index denoting temporal location, with $j=15$ the finest level for this data. Accompanying the coefficient spectrum is its associated energy-scale diagram, a by product of the orthonormal decomposition, containing energy content fractions for each level defined by

$$
\frac{1}{\mathbf{y}^{T} \mathbf{y}} \sum_{k=0}^{2^{j}-1} \gamma_{j k}^{2}, \quad j=J, \ldots, n-1,
$$



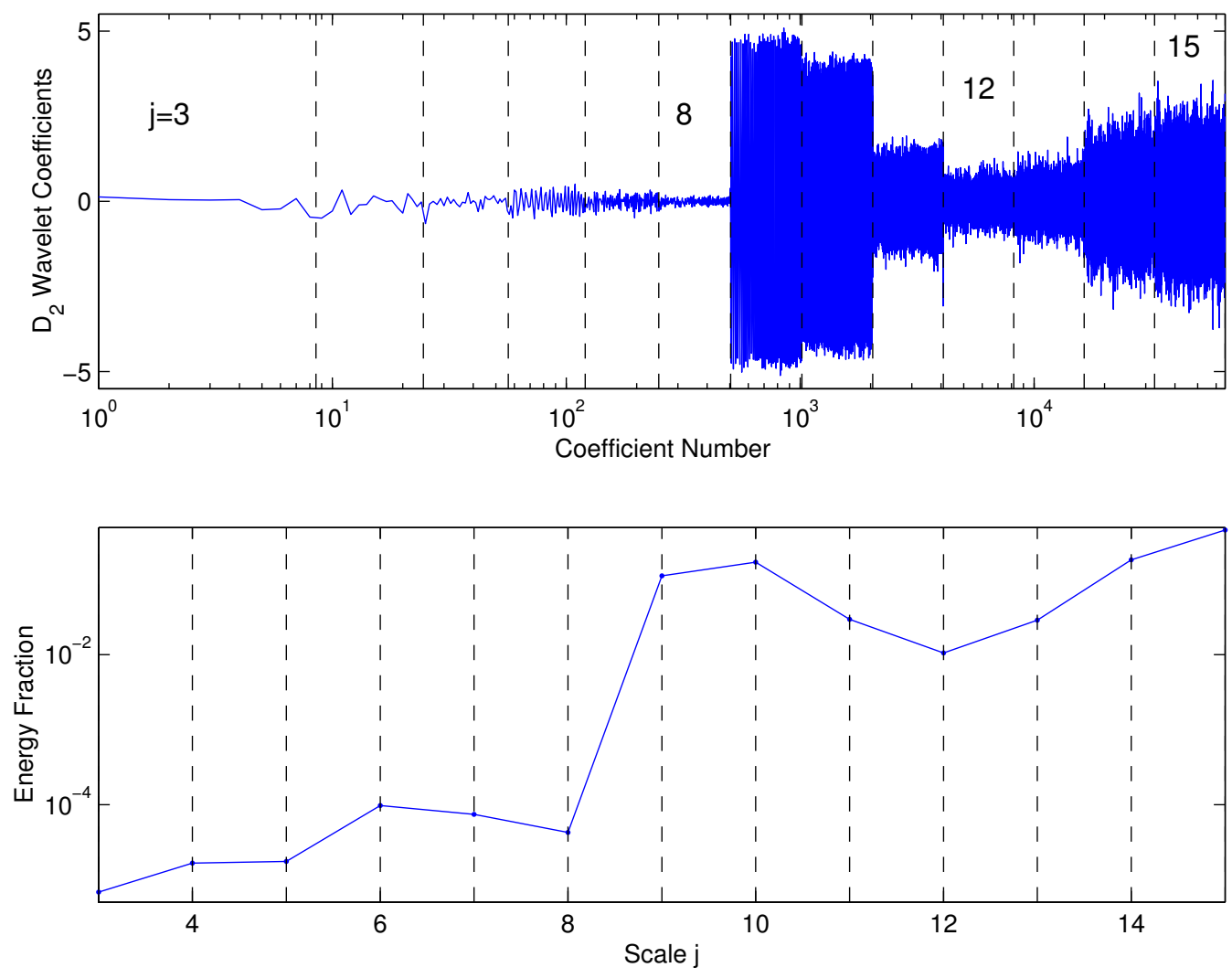

Figure 2: Daubechies $D_{2}$ wavelet coefficient and energy spectra for a flight vibration data sample containing $2^{16}$ points, as represented in the time and frequency domains in Figure 1. Coefficients $\gamma_{j k}$ are arranged level by level in vertical bands, each of which spans the entire data interval on a particular scale. Energy is clearly concentrated on the top 7 scales with a strong contribution from Levels 9 and 10 representing the propeller blade activity. 
which is similar to a frequency spectrum in that evolution information is effectively lost by collapsing each level to a single value. An alternative useful view involves returning to the time domain level by level to generate corresponding components containing the summation of activity on scale $j$ :

$$
\psi_{j}=\sum_{k=0}^{2^{j}-1} \gamma_{j k} \psi_{j k},
$$

or projection of the data onto the level $j$ subspace $W_{j}$, obtained by transform inversion with all coefficients set to zero except those on level $j$.

Initial inspection of Figure 2 indicates a clear division of the spectrum into a strongly dominant group of fine scales featuring relatively large coefficients for $j \geq 9$ and the remaining coarse scales $3 \leq j \leq 8$, with sheer abruptness of the transition between Levels 8 and 9 most noteworthy. In terms of energy, the group of fine scales contain an overwhelming $99.9 \%$ of the total content, reflecting the considerable amounts of high frequency energy seen in the frequency spectrum of Figure 1. An important feature within the fine scales is the pair of adjacent large coefficient blocks on Levels 9 and 10, which generate a corresponding distinct plateau in the energy diagram, beyond which coefficient decay occurs to Level 12 followed by growth across the top 3 levels, where more than two thirds of the energy resides.

\subsection{Propeller blade signatures}

Examination of the signal's $D_{2}$ wavelet level components across all scales, as generated by (2), reveals profound regular features on Levels 9 and 10, generated by the large adjacent coefficient blocks seen in Figure 2, which clearly stand out from their counterparts on the other levels. Close inspection of these components over a period of five shaft rotations in Figure 3 indicates very similar structures on the two levels, each occurring on the shaft rotation scale and shifted in phase by approximately $180^{\circ}$. Superposition of these 

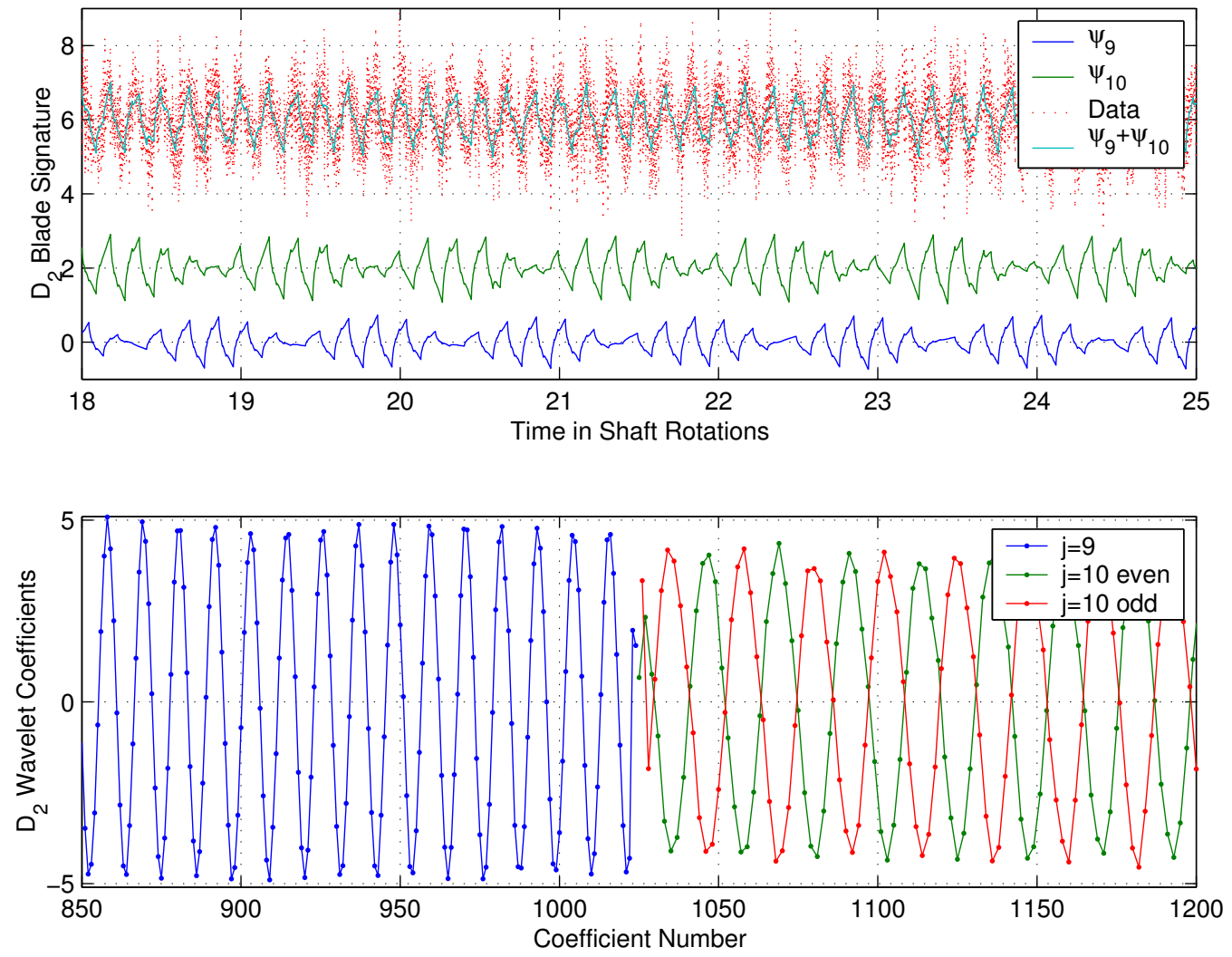

Figure 3: $D_{2}$ level components $\psi_{9}$ and $\psi_{10}$, as generated from (2), showing very similar structure with approximately $180^{\circ}$ phase shift, along with their superposition $\psi_{9}+\psi_{10}$, representing a propeller blade signature in the $D_{2}$ wavelet domain. Corresponding periodic wavelet coefficient patterns that generate these blade signatures are shown in the lower plot, as extracted from the original $D_{2}$ wavelet spectrum in Figure 2. 
two components produces a signal containing six smaller structures over the rotation period, which emerges as an approximation to the blade activity seen in Figure 1, excluding the fine scale spike behaviour. Returning to the coefficient spectra indicates how these structures are formed by particular wavelet combinations, revealing the mechanism by which propeller blade activity is shared between the two adjacent resolution levels. Figure 3 shows a near sinusoidal variation of the Level 9 coefficients, each half cycle of which coincides with a shaft rotation structure, while on Level 10 the even and odd coefficients lie on similar curves, $180^{\circ}$ out of phase, to generate an equivalent structure from finer scale wavelets.

The sharing of blade activity between adjacent resolution levels observed for $D_{2}$ persists for other members of the family, with obvious differences emerging as the key wavelet properties of support width, number of vanishing moments and regularity are simultaneously changed [8]. Figure 4 gives the blade signatures for $D_{2}$ along with those for five other members, clearly showing a tendency for convergence to smooth sinusoidal behaviour with $N$. In the case of Haar, $N=1$, the alternating fine level coefficient patterns produce an effect where almost equal and opposite contributions from successive wavelets yield structures that mimic the coarse level wavelets, with translation. This can be visualised by considering a sequence of consecutive Haar wavelets with equal and opposite coefficients, which yields a shifted sequence of wavelets on the next level. A more subtle difference concerning the sharing between levels also reveals itself upon close consideration of the associated energy diagrams, which display a gradual positive steepening of the segment joining Levels 9 and 10. In addition to this, the adjacent segment connecting Levels 10 and 11 undergoes a more rapid negative steepening, the combined result of which is a sharpening peak on Level 10. Such a sharpening effect is to be expected as the wavelet's frequency resolution increases with support width, and the energy diagram starts to behave like a frequency spectrum. 

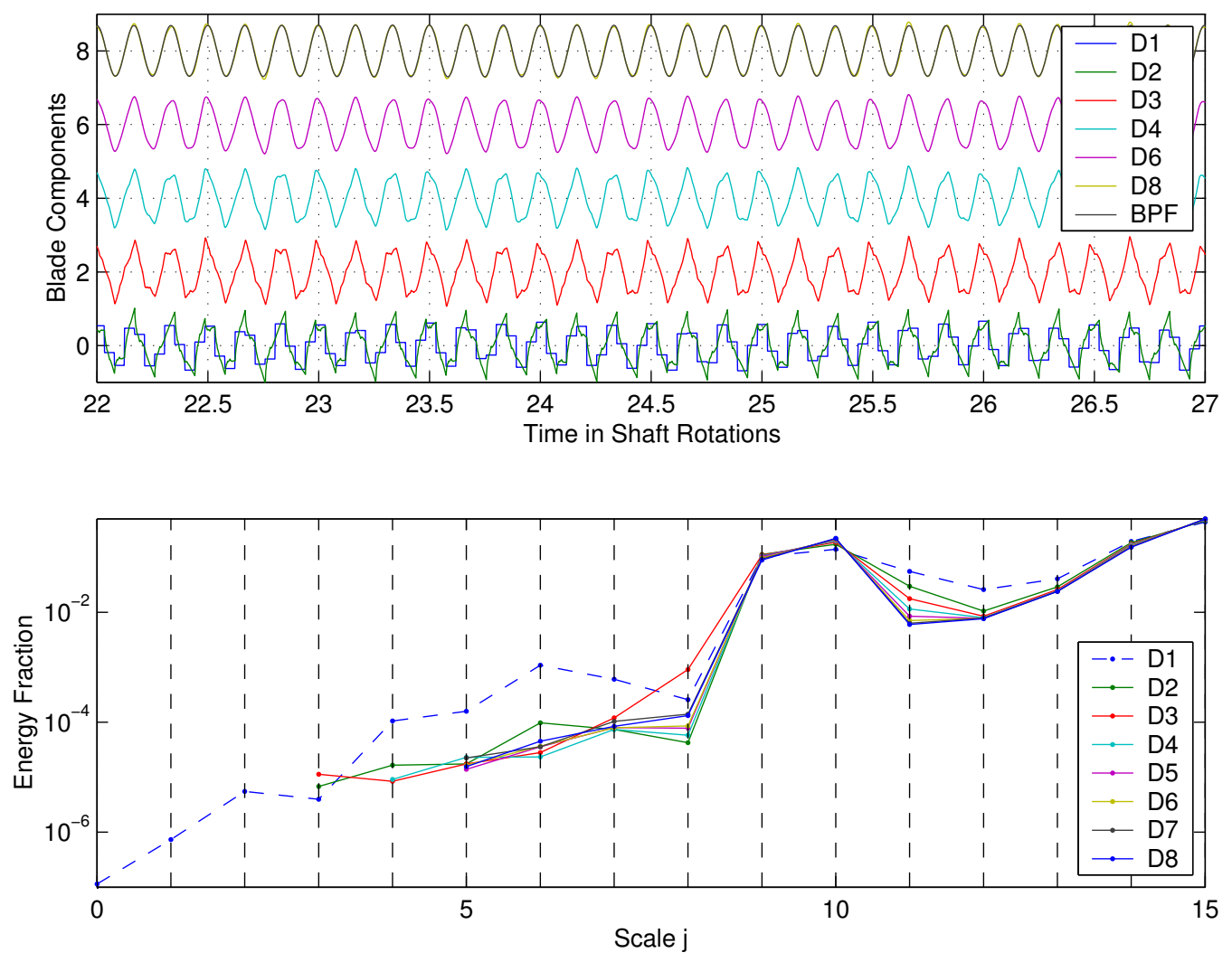

Figure 4: Propeller blade signatures for Daubechies wavelets up to $N=8$ on an interval spanning 5 shaft rotations, exhibiting convergence to smooth sinusoidal behaviour represented by the band pass filtered signal(BPF). The accompanying energy diagrams tend to focus on Level 10 as the wavelet frequency resolution increases with $N$. 

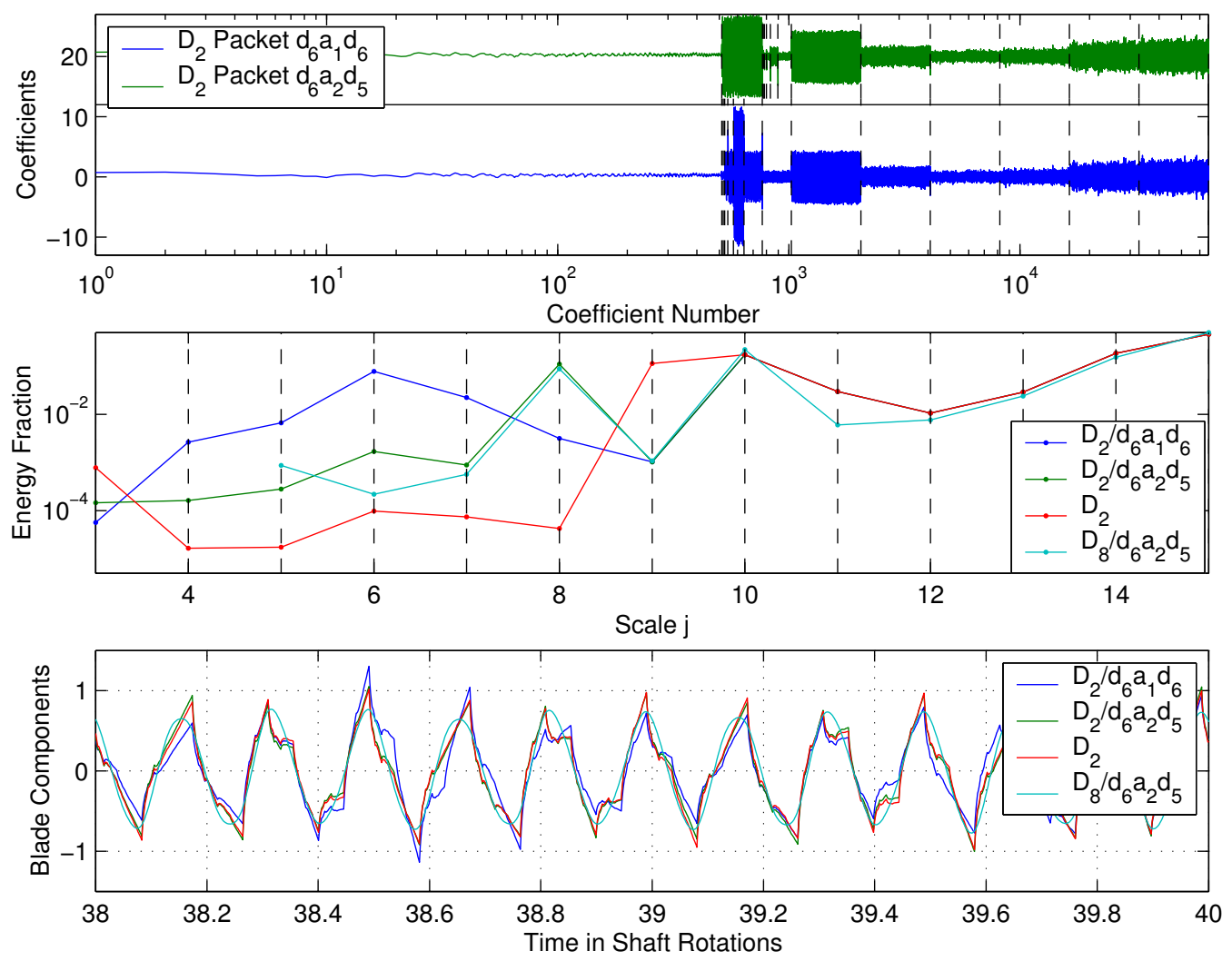

Figure 5: Coefficient and energy spectra for alternative wavelet packet decompositions of the original data in Figure 1. Corresponding blade signatures derived from local energy peaks are also compared in the time domain. 


\subsection{Wavelet packet decomposition}

Discrete wavelet transforms as applied thus far represent a mere single branch of the underlying wavelet packet decomposition tree which includes a vast array of different orthonormal bases in which the data may be represented [6], each possessing a certain resolution pattern in time/frequency space. Removing the restriction of filtering operations to the approximation coefficients at any step and applying the process to the detail coefficients as well considerably broadens the decomposition scope, opening the possibility for optimal basis selection according to some predefined criterion. An example of relevance to propeller blade signatures concerns the isolation of different spectral components from main and tail rotors in helicopter flight vibration data by careful basis selection [5]. The following demonstration will consider some alternative wavelet packet decompositions, with a focus on propeller blade activity and its capture in terms of coefficient and energy distributions.

Consider the dominant coefficient blocks on Levels 9 and 10 in the original $D_{2}$ wavelet spectrum of Figure 2, which provide a blade signature with $2^{9}+$ $2^{10}=1536$ coefficients. Two alternative wavelet packet decompositions, both of which preserve the original $D_{2}$ coefficients down to Level 10, are displayed as coefficient and energy spectra in Figure 5 , labelled $d_{6} a_{1} d_{6}$ and $d_{6} a_{2} d_{5}$. On Level 9, instead of filtering the approximation coefficients these are retained and the detail coefficients are filtered, leaving a local energy minimum on this level. The resulting Level 8 coefficients then share the Level 9 detail energy, with a very large majority of $97.2 \%$ absorbed by the approximation coefficients derived from low pass filtering. For the $d_{6} a_{2} d_{5}$ case these are retained and appear as a strongly dominant block in the spectrum with accompanying energy peak on Level 8, followed by subsequent decay on the remaining levels on which the detail coefficients are retained. In contrast, retaining the detail coefficients on Level 8 for the $d_{6} a_{1} d_{6}$ case spreads the energy across several levels to produce a peak on Level 6 with subsequent decay.

Figure 5 also includes a time domain view showing alternative blade sig- 
natures derived from the local energy peaks of each packet transform. The $d_{6} a_{2} d_{5}$ result, comprising 1280 basis functions from Levels 8 and 10, is closer to the original $D_{2}$ signature in comparison to its $d_{6} a_{1} d_{6}$ counterpart containing 1088 basis functions from Levels 6 and 10. An additional $d_{6} a_{2} d_{5}$ result using the $D_{8}$ filter, possessing similar energy peaks to those of its $D_{2}$ relative, is also shown displaying its expected smoothness.

\section{Summary and conclusions}

An airframe vibration time series data sample has been subjected to various discrete wavelet transforms along with the traditional discrete Fourier transform, with an emphasis on the detection and characterisation of propeller blade activity. The wavelet domain picture immediately indicates a well defined transition separating fine and coarse scales, with over $99.9 \%$ of the signal energy residing in the former region, and approximately two thirds of this on the top three resolution levels. What is seen as a narrow spike in the frequency domain becomes a pair of adjacent large coefficient blocks in the wavelet domain, marking the start of the fine scales, with particular patterns of periodic coefficient activity responsible for generating corresponding blade signatures in the time domain. Repeated calculations across several members of the Daubechies family of orthonormal, compactly supported wavelets display a clear tendency of this blade signature towards smooth sinusoidal behaviour, reflecting enhanced frequency resolution as the wavelet support and smoothness increases. Alternative wavelet packet decompositions demonstrate their ability to focus and capture the blade behaviour with fewer basis functions than required in the standard transform. A remaining important question concerns the very large energy content on the finest scales, or high frequency band, and its physical origin. 


\section{References}

[1] E. Oran Brigham, The Fast Fourier Transform and its Applications, Prentice Hall (1988). C76

[2] A. Cohen, I. Daubechies and P. Vial, Wavelets on the Interval and Fast Wavelet Transforms, Appl. Comp. Harm. Analysis, 1 p54-81 (1993). C79

[3] I. Daubechies, 10 Lectures on Wavelets, SIAM CBMS 61 (1992). C79

[4] P. A. Farrell, C. R. Mouser, D. P. Conser and C. D. Rider, "C-130J-30 Cargo Vibration Flight Test", DSTO TR1310 (2002). C78

[5] M. T. Hale and R. Adhami, Operational Vibration Specification of Helicopter Stores Using Wavelet Analysis, J. of IEST 415 p17-30 (1998). C76, C86

[6] A. Jensen, A. la Cour-Harbo, Ripples in Mathematics, The Discrete Wavelet Transform, Springer (2001). C86

[7] F. C. Moon, Chaotic Vibrations. An Introduction for Applied Scientists and Engineers, Wiley-Interscience (1987). C78

[8] G. Strang and T. Nguyen, Wavelets and Filter Banks, Wellesley Cambridge Press (1997). C76, C79, C83 